ECCOMAS

Proceedia
COMPDYN 2021

$8^{\text {th }}$ ECCOMAS Thematic Conference on Computational Methods in Structural Dynamics and Earthquake Engineering M. Papadrakakis, M. Fragiadakis (eds.)

\title{
METAFOUNDATION AS A NEW APPROACH FOR SEISMIC ISOLATION OF BUILDINGS
}

\author{
S.Fiore $^{1}$, D.Magisano ${ }^{1}$, G.Finocchio ${ }^{2}$, M.Chiappini ${ }^{3}$ \\ ${ }^{1}$ Department of Computer Engineering, Modeling, Electronics and Systems Engineering \\ University of Calabria, Italy \\ stefania.fiore@dimes.unical.it,domenico.magisano@unical.it \\ ${ }^{2}$ Department of Mathematical and Computer Sciences, Physical Sciences and Earth Sciences \\ University of Messina, Italy \\ giovanni.finocchio@unime.it \\ ${ }^{2}$ National Institute of Geophysics and Volcanology \\ Italy \\ massimo.chiappini@ingv.it
}

\begin{abstract}
Metamaterials for seismic protection are engineered to filter the low frequency component of seismic waves. In this study, we investigate the performance of a metastructure focusing on the improvement that the concept design of metamaterials can bring in the field of seismic isolation. The metastructure is realized as composite foundation built as a stack of concrete plates having internal resonant elements. The feasibility of the composite foundation is investigated by performing simulations in which at the base of each column of the building a composite metastructure replaces elastomeric isolator. Dynamic linear analyses are set for concrete multi-story frame buildings by using spectrum-compatible accelerograms for the horizontal components of the ground motion. The main result is the achievement of a reduction in the total displacement of the upper structure with respect a classical base isolation system. This work is a step forward in the understanding of the properties and performance of composite foundations as innovative base isolation system.
\end{abstract}

Keywords: Metamaterials, Seismic isolation. 


\section{INTRODUCTION}

Research and applications in earthquake engineering are focused on seismic protection methodologies for infrastructures and buildings since a lot of decades. Despite the great developments in geophysics seismic monitoring, the incoming earthquake is a dreadful natural event with a significant impact on the structures and people. The reduction of the buildings seismic vulnerability is an important research field in which two main approaches merge; from one side the design capacity strategies based on exploitation of the ductility resources of the building by preserving global safety condition are widely applied in common design activity; from the other side, seismic protection techniques are explored and improved in order to get the best safety condition for the structures when earthquakes occur. Nowadays, seismic protection system is often designed by combining more than one technology, such as elastomeric and damper elements. Within this context, concepts for seismic protection, related to metamaterials, are introduced by researchers as innovative approaches useful at developing technologies for seismic protection.

\section{SEISMIC METAMATERIAL}

Metamaterials attracted interest from the field of physics, where structures that shown negative refraction and permittivity were realized. From the end of the last century research on metamaterials in optical and electromagnetic field exploded [1]. They can be defined as artificial structures with periodic architecture, not available in nature, designed to produce an optimized response to specific excitation. The most interesting property for the development of seismic metamaterials is the chance to exploit the frequency bandgap in which propagation of seismic wave is attenuated [2]. From this starting point a lot of searches in earthquake engineering started and proposed new approach in seismic protection: seismic cloaks and metabarriers as large-scale protection system of large built areas (and ground volume) and metafoundations, as improvement of seismic protection for the single building by including metamaterial design in the foundation structure [3-9]. The main evidence in all the applications is the low frequency working range $(0.1-20 \mathrm{~Hz})$, because both earthquakes frequency content and natural frequencies of most of the building in civil engineering are low. For this reason, seismic metamaterials involve large mass and size.

\section{STANDARD BASE ISOLATION SYSTEM}

Base isolation systems are widely used for seismic protection of concrete multi-story frame buildings. The most popular devices are the elastomeric isolators in which layers of rubber and vulcanized reinforcement steel plates are alternated. Rubber compound guarantees high damping action or other elements with high damping capacity increase this property: the first are called High Damper Rubber Bearings (HDRB), the second Lead Rubber Bearings (LRB). The main features of elastomeric devices are the horizontal low stiffness and vertical load capacity need to transfer the entire dead and live load to the foundation. When this devices are installed at the base of the building, mechanical decoupling between superstructure and ground is achieved and, the vibration period of the structure shifts to higher period and the total acceleration of structural mass is reduced. Their effectiveness is tested by massive experimental tests and, by now, thousands of isolated buildings with this system demonstrate good performance when seismic event occurs. 


\section{METASTRUCTURE}

From analytical point of view, the study of seismic metamaterials is manly based on the theory of phononic crystals and periodic chains of mass-in-mass system. The bandgap computed with the dispersion relation defines the attenuation regions of metamaterial in the space frequency-wavenumber [10]. Metastructure is proposed in this work, to replace elastomeric devices in standard base isolation system. In this study metamaterial is characterized by external element with inclusions therefore simplified lumped model is a bi-atomic chain or mass-inmass chain. The parameters of the model are: mass of external element $\left(\mathrm{m}_{1}\right)$, mass of internal element $\left(\mathrm{m}_{2}\right)$, stiffness connection between consecutive external elements $\left(\mathrm{k}_{1}\right)$ and stiffness connection between internal and external elements $\left(\mathrm{k}_{2}\right)$. The dispersion relation of the periodic chain of the mass-in-mass system is given by:

$$
m_{1} m_{2} \omega^{4}-\left(k_{2}\left(m_{1}+m_{2}\right)+2 m_{2} k_{1}(1-\cos (k L))\right) \omega^{2}+2 k_{2} k_{1}(1-\cos (k L))=0
$$

Starting point of this study are the results obtained by experimental tests on a demonstrator built of four stacked concrete square plate in each of which five cylindric steel resonators are allocated [11]. Connections was realized by rubber elements excited in tension-compression regime. The demonstrator was tested under dynamic loading with sinusoidal input, from 1 to $8 \mathrm{~Hz}$, applied at the bottom plate. Measurements of displacements confirm a bandgap near 5 Hz. From the bottom to the top, input signal is trapped, and the top plate does not move. Demonstrator was designed with external mass $\left(\mathrm{m}_{1}\right)$ equal to $317 \mathrm{~kg}$, total internal mass $\left(\mathrm{m}_{2}\right)$ equal to $245 \mathrm{~kg}$, external stiffness connection $\left(\mathrm{k}_{1}\right)$ equal to $155 \mathrm{e} 3 \mathrm{~N} / \mathrm{m}$ and internal stiffness $\left(\mathrm{k}_{2}\right)$ connection equal to $1080 \mathrm{e} 3 \mathrm{~N} / \mathrm{m}$. This values are used in the following, as reference in design of metastructure simulated in this work.

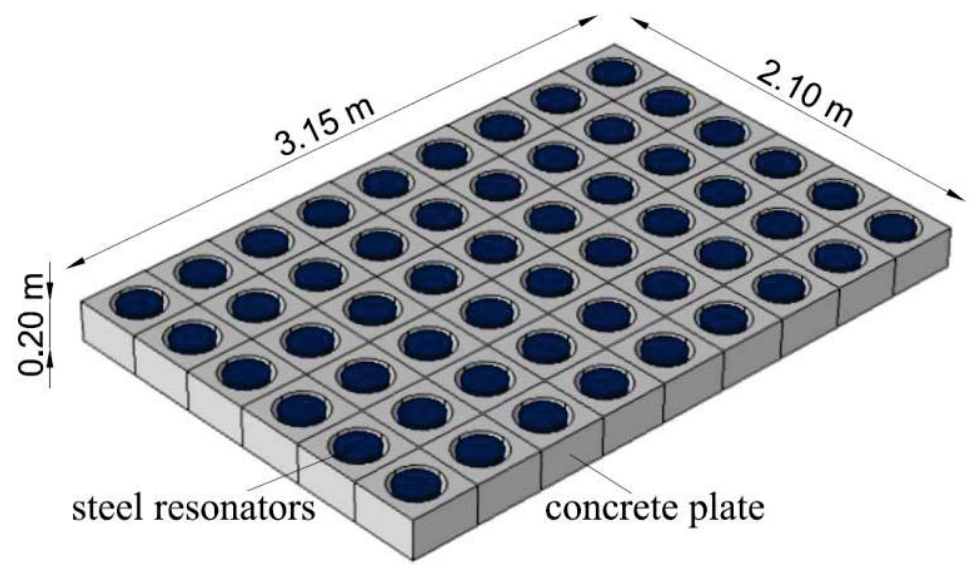

(a)

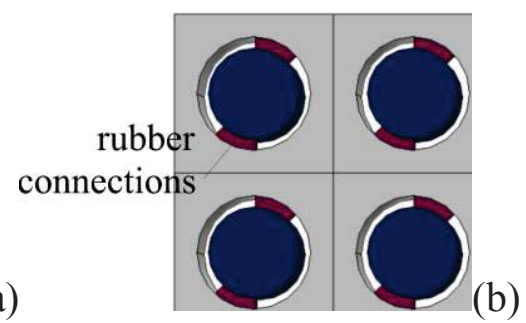

Figure 1: (a) geometry of one plate of the metastructure designed in this work with values shown in Tab 1. (b) details about rubber connection between resonators and concrete plate.

\section{NUMERICAL INVESTIGATION}

In this part, seismic performance of a square plan concrete four-story frame building is studied An artificial seismic signal is applied and the results between the performance of the proposed metafoundation and the standard base isolation are compared. The building has a symmetrical geometry, with side equal to $6 \mathrm{~m}$, height of each story equal to $3.5 \mathrm{~m}$, section column of $0.5 \times 0.5 \mathrm{~m}$, section beam of $0.4 \times 0.6 \mathrm{~m}$. Concrete floors have thickness equal to 0.2 
$\mathrm{m}$ and base floor, that ensure rigid motion of the isolated structure, $0.35 \mathrm{~m}$. The dead load is estimated at $7 \mathrm{kN} / \mathrm{mq}$ while live load is set at $2 \mathrm{kN} / \mathrm{mq}$.

The design of metastructure is performed in order to define the lower branch of the bandgap by keeping appropriate horizontal stiffness and by limiting structural mass. Reference design is the experimental device [11] whose plate can be used as modular element and in this work by considering six plate in $2 \times 3$ array. Fig. 1 shows sketch of one plate of the metastructure while Tab. 1 collects geometrical and mechanical data: $\mathrm{m}_{1}$ is the concrete plate mass, $\mathrm{m}_{2}$ is the total mass of resonators, $\mathrm{k}_{2}$ is the liner compression/traction stiffness of rubber elements that connect resonators with concrete plate, while $\mathrm{k}_{1}$ is the horizontal stiffness between overlapped plates that is realized by appropriate connection elements. Theoretical expected dispersion relation obtained by using this design is shown in Fig.2.

\begin{tabular}{cccc}
\hline $\mathrm{m}_{1}$ & $\mathrm{k}_{1}$ & $\mathrm{~m}_{2}$ & $\mathrm{k}_{2}$ \\
\hline $1902 \mathrm{~kg}$ & $0.31 \mathrm{e} 6 \mathrm{kN} / \mathrm{mm}$ & $1470 \mathrm{~kg}$ & $1.08 \mathrm{e} 6 \mathrm{kN} / \mathrm{mm}$ \\
\hline
\end{tabular}

Table 1: Mechanical lumped parameters of metastructure.

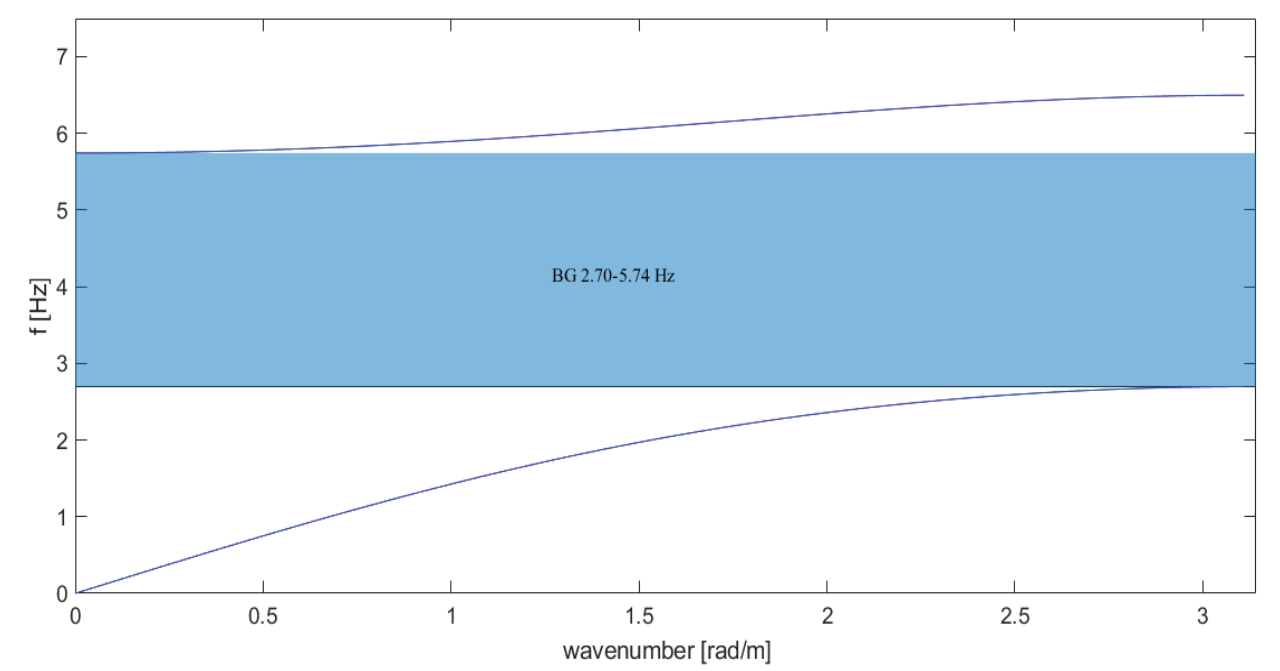

Figure 3: Dispersion curves of mass-in-mass chain with lumped parameters equal to data in Table 1. Theoretical expected bandgap starting at $2.70 \mathrm{~Hz}$.

In order to compare the behavior of metastructure and standard base isolation with elastomeric devices (named BI in the following for brevity), equivalent linear stiffness for BI is assumed qual to $\mathrm{k}_{2}$.

\subsection{Artificial seismic input}

Dynamic loading applied at the building is a set of artificial ground acceleration timehistory computed through the software SIMKE_GR (SIMulation of earthQuaKE ground motions) [12]. Indeed, in compliance with construction standards in design of structures for earthquake resistance (European codes and Italian national codes), seismic analyses can be performed by using a set of spectrum-compatible artificial accelerograms at elastic response spectra with a viscous damping equal to $5 \%$. The source data of artificial earthquakes generation are collected in Table 2 and one of the accelerograms is shown in Figure 3. Accelerograms concern horizontal component of the ground motion while vertical one is neglected. 


\begin{tabular}{ccccc}
\hline site & $\begin{array}{c}\text { design } \\
\text { life }\end{array}$ & $\begin{array}{c}\text { ground } \\
\text { type }\end{array}$ & ag/g & $\begin{array}{c}\text { viscous } \\
\text { damping }\end{array}$ \\
\hline Messina (IT) & 50 & $\mathrm{D}$ & 0.337 & 0.05 \\
\hline
\end{tabular}

Table 2: Spectrum-data for the generation of the artificial horizontal seismic accelerograms.
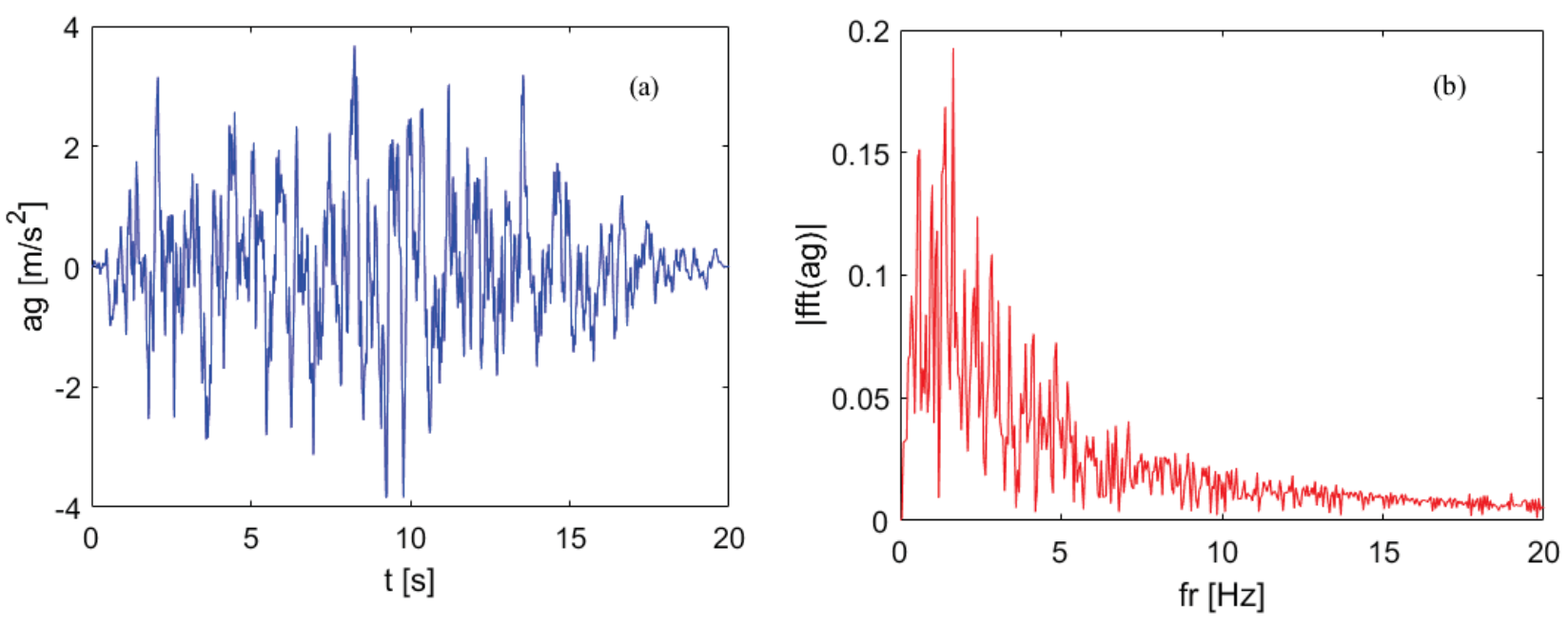

Figure 3: Artificial spectrum-compatible earthquake (a) time-history ground acceleration, (b) ground acceleration in frequency represented by Fourier amplitude spectrum.

\subsection{Dynamic analysis: model}

The building is modeled by using the structural software SAP2000 [13]. A diaphragm constraint is applied at each story to model high stiffness of the concrete floor. Metastructure is modeled by using a simplified configuration of points (one for each plate) connected by mechanical link with linear force-displacement law. Damping in metastructure is neglected in order to observe mechanical response associated with the design configuration of mass and stiffness. Linear dynamic analysis is performed by using direct integration method on Eq. 2 that represents the linear system of equations of motion of the building and metastructure (coupled) in which $M$ is the stiffness matrix, $C$ the viscous damping matrix, $K$ the stiffness matrix, $\ddot{u}, \dot{u}$ and $u$ are acceleration, velocity and displacement fields and $\ddot{u}_{g}$ is the ground acceleration. Vertical motion is neglected in metastructure. Viscous proportional damping is set equal to $5 \%$ at 1 and $10 \mathrm{~Hz}$.

$$
M\left(\ddot{u}+\ddot{u}_{g}\right)+C \dot{u}+K u=0
$$

In addition to artificial earthquake, a sweep signal, modulated between 0.1 and $10 \mathrm{~Hz}$, is applied at the base of the structure to check working frequency range of metastructure and frequency response function is evaluated as ratio between power spectral density of displacement field of the check point and that of input as shown in Eq.3.

$$
F R F=20 \cdot \log _{10} \frac{\operatorname{PSD}\left(u_{T}\right)}{\operatorname{PSD}\left(u_{q}\right)}
$$

Results show comparison between response of structure with fix base, standard elastomeric devices and metastructures. 


\subsection{Results}

The total displacement at the top of the building is shown in Fig. 4. Total displacement for isolated structures is almost entirely a rigid motion of the whole structure respect to the plane of the isolation system because bending modes are attenuated. Reduction of displacement when metastructure is set (red line) can be observed in the most time of structural response, in some points is closed at 50\% compared to the BI case. This result improves performance in seismic protection because amplitude and number of oscillations of the superstructure is reduced. Fig. 5 shows the frequency response function of metastructures and highligths reduction at lower frequency compared to BI while peaks between 1 and $3 \mathrm{~Hz}$ concern local modes of the metastructure (motion in-phase and out-phase between plate and resonators).

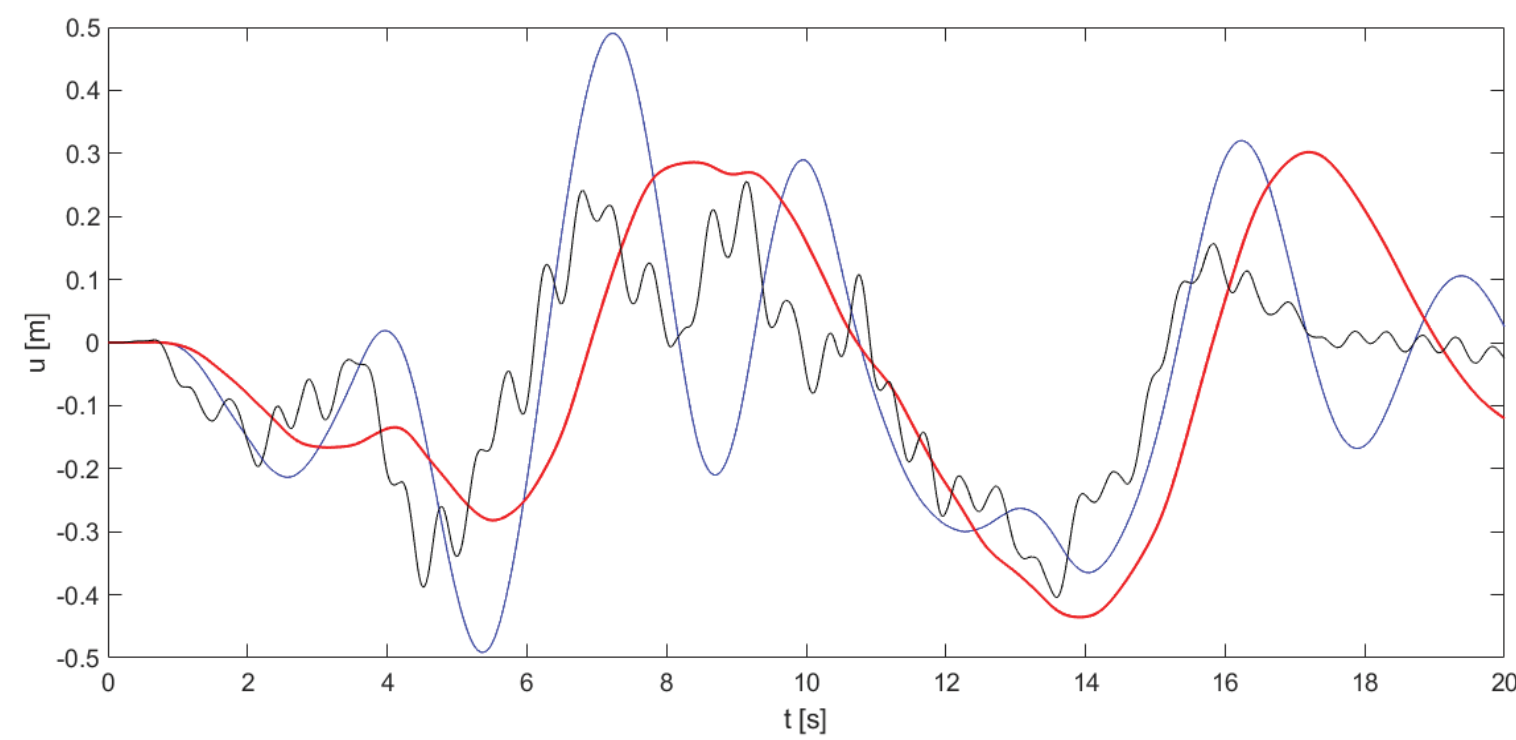

Figure 4: Time-history total displacement at the top of the building $\left(\mathrm{u}_{\mathrm{T}}\right)$ with fix base (black line), BI (blue line), metastructure (red line) by applying artificial earthquake $\left(\mathrm{ug}_{\mathrm{g}}\right)$.

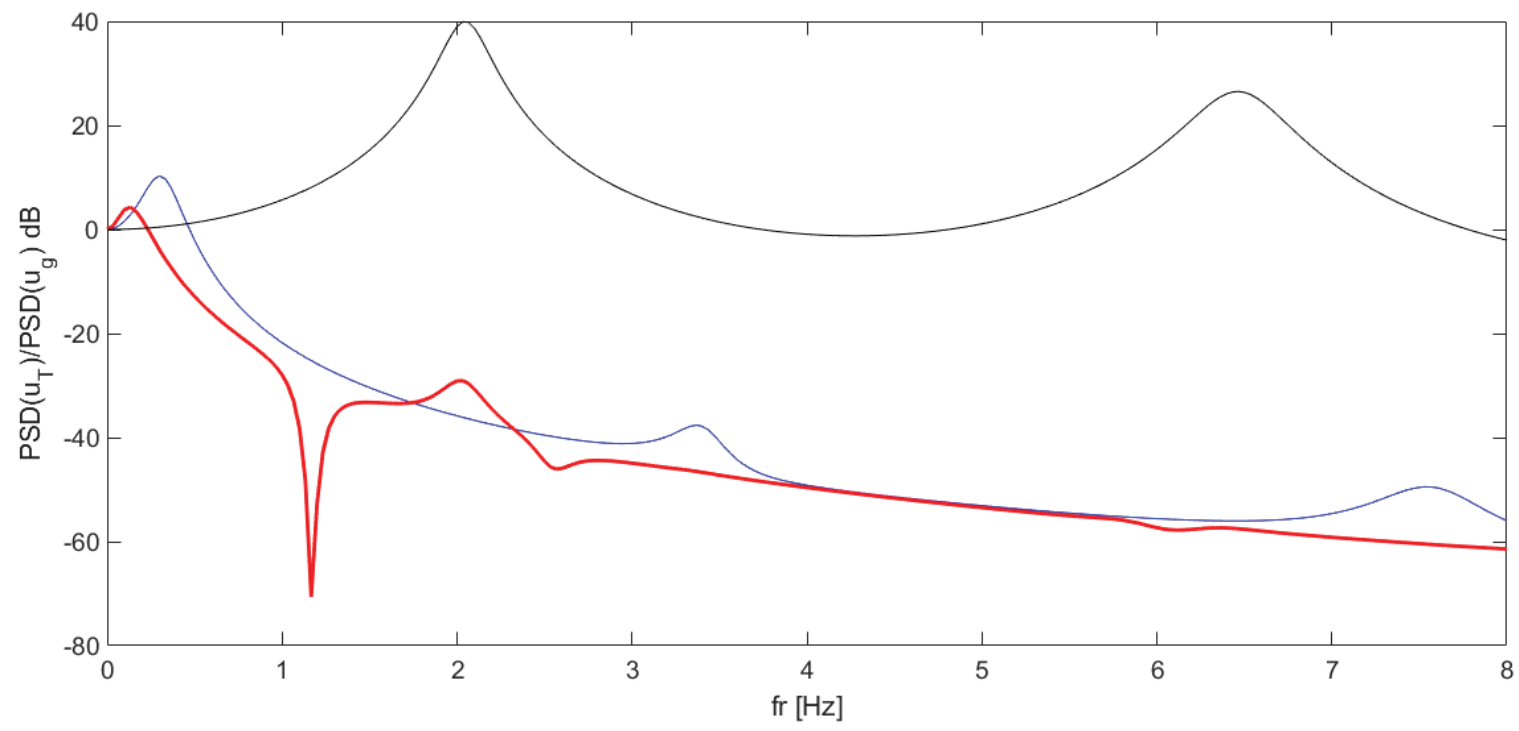


Figure 5: Frequency Response Function evaluated as ratio between power spectral density of total displacement at the top of the building $\left(\mathrm{u}_{\mathrm{T}}\right)$, with fix base (black line), BI (blue line), metastructure (red line), and ground motion $\left(\mathrm{u}_{\mathrm{g}}\right)$ set as modulated sweep signal.

\section{CONCLUSIONS}

- One metastructure characterized by four overlapped plates with inside resonators is proposed as innovative system od base isolation, by starting from good results in experimental investigation;

- The goal of metastructures would be the total isolation of the structure during earthquake but tuning bandgap at very low frequencies is very hard because requires lower stiffness and higher masses;

- By setting appropriate configuration of metastructure bandgap closed to $2 \mathrm{~Hz}$ is obtained and filter effect is enforced compared to standard BI system;

- Good reduction in oscillations and amplitude total displacements of four-story frame building under seismic dynamic loading is provided. This can have important benefits for the occupant because perception of earthquake is reduced.

\section{ACKNOWLEDGEMENT}

This work has been supported by MARIS scrl (https://www.maris-ricerca.it/) and by the Project (Progetto Premiale) "Strategic Initiatives for the Environment and Security -SIES". The authors acknowledge the discussions and support in learning SAP from the research group in structural mechanical and metamaterials of Professor Eleni Chatzi at ETH Zurich, particularly Dr. Andrea Colombi, Dr. Giulia Aguzzi and Dr.Panagiotis Martakis.

\section{REFERENCES}

[1] T.J. Cui,D.R. Smith,R,Liu, Metamaterials, Theory, Design and Applications, Springer, 2010.

[2] D. Mu, H.Shu, L.Zhao, S. An; A Review of Research on Seismic Metamaterials, Advanced engineering materials (2019).

[3] A.O. Krushynska, M. Miniaci, F. Bosia, N.M. Pugno, Coupling local resonance with Bragg band gaps in single-phase mechanical metamaterials, Extreme Mechanics Letters 12 (2017) 30-36.

[4] Jing Bao, Zhifei Shi, Hongjun Xiang, Dynamic Responses of a Structure with Periodic Foundations, Journal of Engineering Mechanics, Vol.138 Issue 7 (2012).

[5] Y. Zeng, Y. Xu, K. Deng, P.Peng, H.Yang, M. Muzamil, Q. Du, A broadband seismic metamaterial plate with simple structure and easy realization, Journal of Applied Phyics ,125, 224901 (2019).

[6] Z.Chenga, Z.Shia, A.Palermo, H.Xianga, W. Guoc, A. Marzani, Seismic vibrations attenuation via damped layered periodic foundations, Engineering Structures 211 (2020) 110427. 
[7] V. Dertimanis,I.A. Antoniadis, E.N. Chatzi, Feasibility Analysis on the Attenuation of Strong Ground Motions Using Finite Periodic Lattices of Mass-in-Mass Barriers, J. Eng. Mech., 2016, 142(9): 04016060

[8] X.H. Shen, C.T. Sun, M.V.Barnhart, G.L.Huang, Analysis of Dynamic Behavior of the Finite Elastic Metamaterial-Based Structure With Frequency-Dependent Properties, Journal of Vibration and Acoustics, June2018, Vol. 140 / 031012-1.

[9] A.Colombi, R.Zaccherini, G.Aguzzi,A.Palermo,E.Chatzi Mitigation of seismic waves: metabarriers and metafoundations bench tested, Journal of Sound and Vibration, Volume 485, 27 October 2020, 115537.

[10] L. Brillouin, Wave propagation in periodic structures, Dover (1953).

[11] O. Casablanca, G. Ventura, F. Garescì, B. Azzerboni, B. Chiaia, M. Chiappini, and G. Finocchio, Seismic isolation of buildings using composite foundations based on metamaterials, Journal of Applied Physics 123, 174903 (2018).

[12] Gelfi, P. 2009. SIMQKE_GR, Artificial earthquakes compatible with response spectra, software, Internet site: http://dicata.ing.unibs.it/gelfi/software/simqke/simqke_gr.htm.

[13] CSI Analysis Reference Manual for SAP2000,ETABS,SAFE and CSiBridge (2017). 